\title{
Synthesis and Study of ternary copolymerized cationic polyacrylamide
}

\author{
Haidi Cai ${ }^{1, a}$, Fangyou Meng ${ }^{1, b}$, Changhong $\mathrm{Li}^{2, \mathrm{c}}$, Feng Zhao ${ }^{1, \mathrm{~d}^{*}}$ \\ ${ }^{1}$ Jiangxi Key Laboratory of Organic Chemistry, Jiangxi Science \& Technology Normal University, \\ Fenglin Street Nanchang, jiangxi 330013, China. \\ ${ }^{2}$ Changjiu Agrochemical Co., Ltd, Nanchang, jiangxi 330012,China.

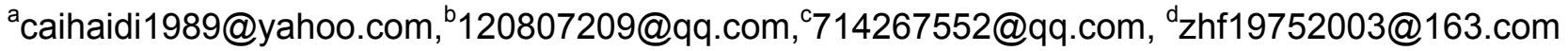

Keywords: ternary copolymerized, cationic polyacrylamide, Aqueous solution polymerization.

\begin{abstract}
The ternary cation flocculant P(AM-DMDAAC-DMC) was synthesized by aqueous solution polymerization with raw materials of acrylamide(AM),and dimethyl diallyl ammonium chloride(DMDAAC) and methacryloyloxyethyltrimethyl ammonium chloride(DMC) with the complex redox as initiator.Effects of cationic mononer ratio,mononer mass fraction, the amount of initiator and reaction temperature on the viscosity of the products were investigated. The results indicate that the viscosity of the copolymer can reach $10.26 \mathrm{dL} / \mathrm{g}$, and the terpolymer demonstrates excellent water-solubility.The chemical structure was investigated by IR.
\end{abstract}

\section{Introduction}

Polyacrylamide, which is a linear macromolecule polymer(CPAM), is a general designation of acrylamide homopolymer and copolymer obtained from acrylamide and other monomers. Cationic polyacrylamide having negatively charged particles in water can act as a "charge neutralization" and "adsorption bridging" role, at the same time, he can effectively flocculation and to strengthen the solid-liquid separation process, etc. ${ }^{[1]}$, so it can be used as polymer flocculants are widely used in oil exploration, paper making, metallurgy, mining, textile, defense, household chemicals, water treatment and other fields ${ }^{[2-3]}$.In order to obtain a particular purpose cationic polyacrylamide product,we usually use a cationic monomer with acrylamide monomer to get ${ }^{[4]}$.Commonly used cationic monomers are diallyl dimethyl ammonium chloride (DMDAAC), methacryloyloxyethyltrimethyl ammonium chloride (DMC), etc. ${ }^{[5-7]}$ In this paper, DMC, DMDAAC and AM were terpolymers, and its polymerization conditions were studied.

\section{Experimental}

\section{Materials and Instrument.}

The AM was purchased from Damao (Tianjin, China);dimethyl diallyl ammonium chloride(DMDAAC) and methacryloyloxyethyltrimethyl ammonium chloride(DMC) was purchased from Aladdin;Ammonium peroxydisulfate used as the oxidant was provided by Yongda (Tianjin, China);Ammonium iron(II) sulfate used as reducing agent was provided by Aladdin.

\section{Experimental method.}

The desired amount of acrylamide and other additive was weighed out and transferred to jars; The jar was sealed with nitrogen in addition to oxygen,A syringe was used to transfer the desired amount of initiator into the sealed jars containing the monomer; After the polymerization reaction was finished, the reaction jars was opened and the polymer was removed out into a beaker containing acetone then the mixture was standing for some time. The resulting products were dried and crushed.

\section{Characterization.}

Determination of viscosity: The viscosity of polymer was measured by Ubbelohde viscometer.

Structure characterization: The structural characteristics of the product was measured by FTIR Spectrometer Two. 


\section{Results and Discussion}

Effect of monomer mass fraction on the viscosity of products.

The choice of monomer mass fraction plays a vital role in polymerizing acrylamide. This paper studies the effect of monomer mass fraction on the viscosity of products. It is shown in Fig.1.

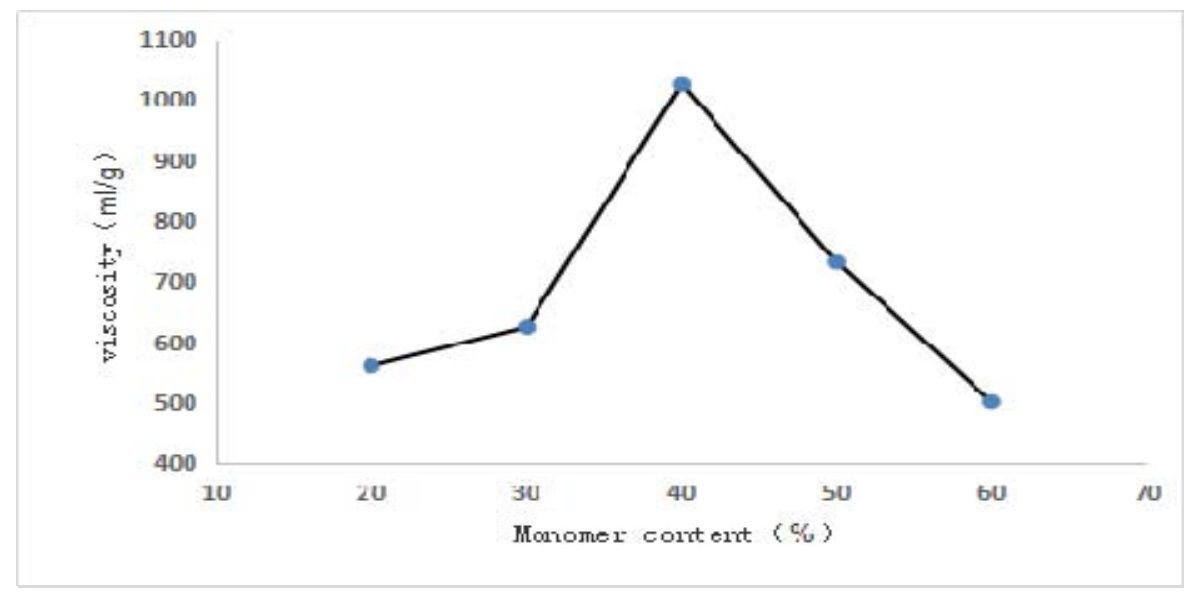

Fig.1 Effect of monomer mass fraction on the viscosity of products

As can be seen,form Fig.1, with the monomer concentration increases, the molecular weight of the copolymer is first increases and then decreases when the mass fraction of $40 \%$, its molecular weight is maximized. This is because: With the monomer concentration increases, the rate of polymerization increases, the degree of polymerization increases, the viscosity increases. However, the polymerization reaction is an exothermic reaction, the polymerization rate continued to increase may cause a temperature increase of the reaction system, resulting in a chain transfer reaction rate is accelerated, the molecular weight decreased.Therefore, we chose $40 \%$ as the best mass fraction of the monomer.

Effect of mononer ratio on the viscosity of products.

Fixed monomer mass fraction is $40 \%$, and other polymerization conditions remain unchanged, the quality of investigation monomer ratio on the intrinsic viscosity of the copolymer, and the results shown in Table 1.

Table 1.Effect of mononer ratio on the viscosity of products

\begin{tabular}{cccc}
\hline $\begin{array}{c}\mathrm{m}(\mathrm{AM}): \mathrm{m}(\mathrm{DMDA} \\
\mathrm{AC}): \mathrm{m}(\mathrm{DMC})\end{array}$ & Solubility & Viscosity $(\mathrm{ml} / \mathrm{g})$ & Conversion rate $(\%)$ \\
\hline $5: 4: 1$ & & & 88 \\
$5: 3: 2$ & good & 515 & 89 \\
$5 ; 2.5 ; 2.5$ & good & 576 & 90 \\
$5: 2 ; 3$ & good & 687 & 92 \\
$5: 1: 4$ & good & 784 & 95 \\
\hline
\end{tabular}

As it can be seen from Table 1, when the proportion of AM unchanged, the intrinsic viscosity of terpolymer product quality increases when the mass of DMC increases, indicate that the reactivity of DMC was larger than DMDAAC.Therefore, we choose the best mass ratio of monomer to $\mathrm{m}$ $(\mathrm{AM}): \mathrm{m}(\mathrm{DMDAAC}): \mathrm{m}(\mathrm{DMC})=5: 1: 4$.

Effect of the amount of initiator on the viscosity of products.

The viscosity of polymer for the reactions performed at initiator concentration are shown in Fig.2 


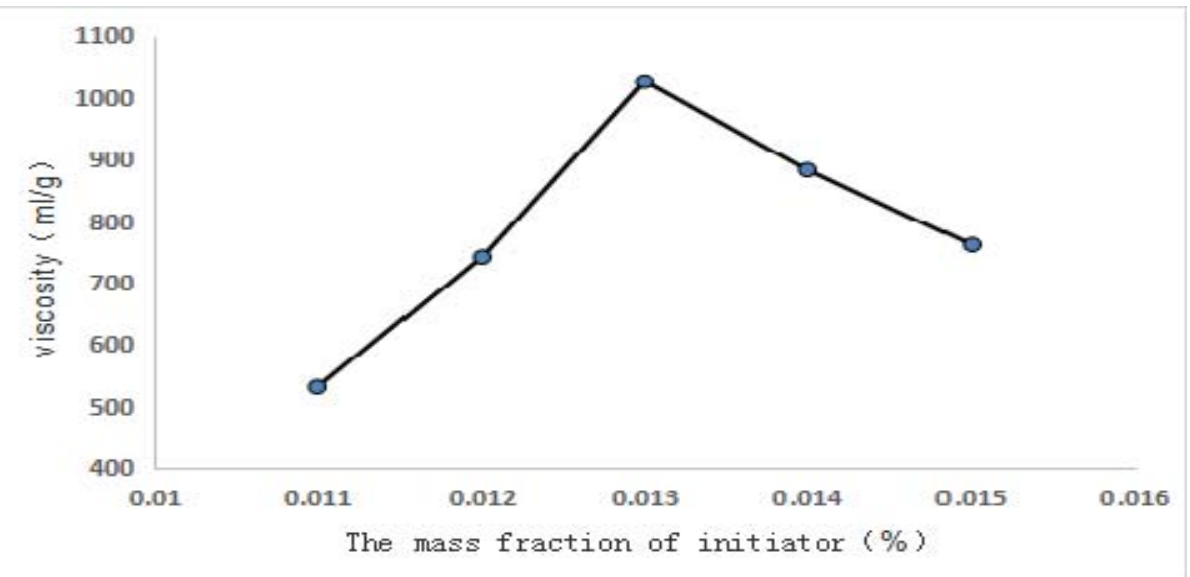

Fig.2 Effect of the amount of initiator on the viscosity of products

We use the complex redox initiator to initiate the reaction, enabling more efficient initiator, water-soluble products better. As can be seen from Fig.2, the viscosity of the copolymer with the mass fraction of initiator first increases and then decreases, when the mass fraction of initiator agent is $0.013 \%$, the characteristics of the copolymer viscosity maximum for $10.26 \mathrm{dl} / \mathrm{g}$.

Effect of the reaction temperature on the viscosity of products.

The reaction temperature is one of the key factors affecting the polymerization reaction, its impact on the intrinsic viscosity of the product is more significant.As is shown in Fig.3

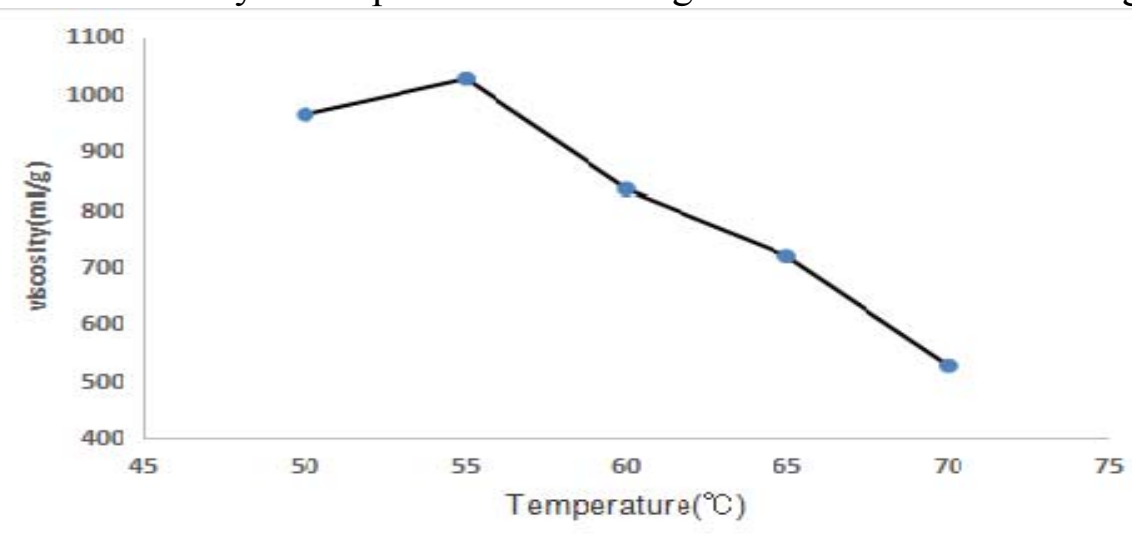

Fig.3 Effect of the reaction temperature on the viscosity of products

It is clear that from Fig.3,Polymerization rate increase with reaction temperature as a result of increase in the decomposition rate constant. After reaching the maximum value, the polymerization rate was decreased with higher of reaction temperature. The figure that the polymer viscosity increased first and then decreased. When the reaction temperature is $55{ }^{\circ} \mathrm{C}$, its intrinsic viscosity is maximized.This is because when a lower reaction temperature, the initiator decomposition rate is low, a small number of radical generation, resulting in the polymerization reaction is slow and incomplete, reducing the viscosity of the copolymer. When the reaction temperature is too high, lead to faster, while the chain transfer reaction rate also accelerated, resulting in reduced viscosity of the copolymer.

FTIR spectrum of ternary copolymer P(AM-DMC-DMDAAC).

Structural spectrograms are shown in Fig.4. 


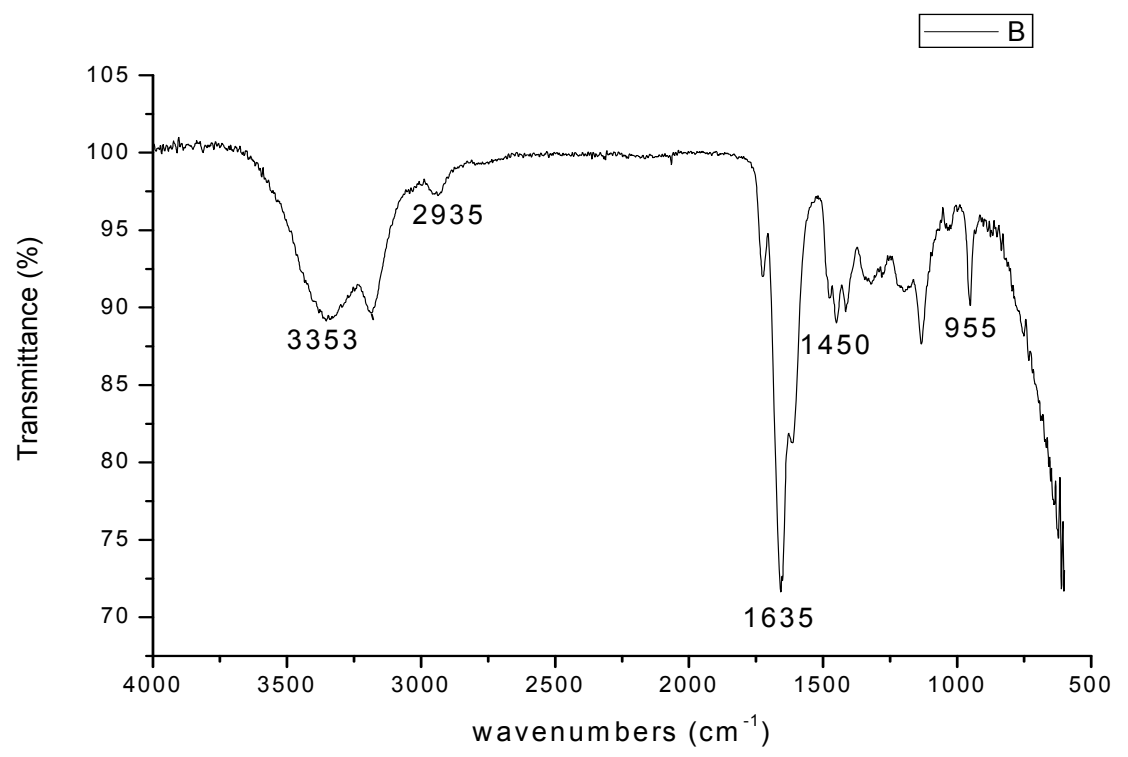

Fig. 4 Infrered of P(AM-DMC-DMDAAC)

The peak value of vibrating absorption of amidogen $\left(-\mathrm{NH}_{2}\right)$ is $3353 \mathrm{~cm}^{-1}$, and carbonyl of acylamino group $1635 \mathrm{~cm}^{-1}$. The stretching vibration peak at $2935 \mathrm{~cm}^{-1}$ is associated with the $\mathrm{C}-\mathrm{H}$ bond in the backbone of the copolymer, and $1450 \mathrm{~cm}^{-1}$ is assigned to the unsymmetrical bending vibration absorption peak of methyl bonded with $\mathrm{N}^{+}$. The absorption peaks at $955 \mathrm{~cm}^{-1}$ are respectively attributed to the bending vibration peak for methylene and the stretching vibration absorption peak for quaternary ammonium $\left(-\left(\mathrm{CH}_{2}\right)_{2} \mathrm{~N}\left(\mathrm{CH}_{3}\right)_{3} \mathrm{Cl}\right)$.According to the FTIR analysis, the results confirmed the polymer was synthesized.

\section{Summary}

Aqueous solution polymerization technique was used to synthesis ternary copolymerized cationic polyacrylamide.In this study,the optimum synthetic conditions can be achieved according to the mononer ratio is $\mathrm{m}(\mathrm{AM}): \mathrm{m}(\mathrm{DMDAAC}): \mathrm{m}(\mathrm{DMC})=5: 1: 4$, the monomer mass fraction is $40 \%$,initiator mass fraction is $0.013 \%$, the polymerization temperature is $55^{\circ} \mathrm{C}$, the viscosity of the polymer obtained in this condition reaches $10.26 \mathrm{dL} / \mathrm{g}$, and has good water solubility.

\section{Acknowledgements}

The authors acknowledge financial support by the Nature Science Foundation of Jiangxi Province (20132BAB206032), and the Project of the Science Funds of Jiangxi Education Office(KJLD14068), and Jiangxi Science and Technology Normal University Key Laboratory of Organic (Key training base).

\section{References}

[1] SI Xiaohui, YUE Qinyan, GAO Baoyu , et al.Synthesis of P(DMDAAC-AM)as cationic polymeric flocculants by dispersion polymerization[J] .Journal of Shandong University :Natural Science, 2008, 43(1):28-32.

[2] SHANG Hongzhou, LIU Jianping, ZHENG Yubin .Advances in cationic poly acrylamide polymers[J] .Modern Chemical Industry, 2007, 27(1):118-121.

[3] ZHANG Long, LIU Xueyan, LI Changhai, et al .Synthesis of acrylamide-2-acryloxyethytrimethyl ammonium chloride copolymer and its 
flocuulation[J] .Polymer M aterials Science \&Engineering , 2002, 15(6):59-61.

[4] SUN Yanxia, CHE Jitai .Development of high molecular weight acrylamide-DAC copolymer[J] .Advances in Fine Petrochemicals, 2003 , 4(9):44-45.

[5] Yoshio Mori. Blended amphoteric PAMs: High performance as retention aids [J].Tappi Journal. 2007:17-20.

[6] Zhu J F, Zhang G H, et al. Preparation of Amphoteric Polyacrylamide Flocculant and Its Application in The Teatment of Tannery Wastewater[J].Journal of applied polymer science. 2011,120(1):518-523.

[7] Rivas B L, Pereira E D, Palenica M, et al. Water-soluble functional polymers in conjunction with membranes to remove pollutant ions from aqueous solutions[J]. Progress in Polymer Science. 2010,664:29. 